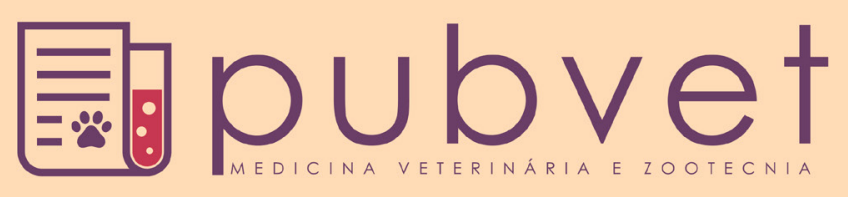

HTTP://DX.DOI.ORG/10.22256/PUBVET.V11N6.561-565

\title{
Aspectos da qualidade físico-química em filé de Carapeba (Diapterus rhombeus) minimamente processada
}

\author{
Bárbara Camila Firmino Freire ${ }^{1 *}$, Karoline Mikaelle de Paiva Soares ${ }^{2}$, Vilson Alves de \\ Góis $^{3}$, Antônio Cleyton Arruda de Azevedo Costa ${ }^{4}$, Daniela Rayane da Silva Morais ${ }^{5}$, \\ Amanda Sabino de Souza ${ }^{6}$
}

${ }^{I}$ Mestranda em Ambiente, Tecnologia e Sociedade. Centro de Ciências Agrárias, Universidade Federal Rural do Semi-Árido. Mossoró-RN, Brasil.bcamila.ffreire@gmail.com

${ }_{2}^{2}$ Professora do Programa de Pós-Graduação em Ambiente, Tecnologia e Sociedade, Universidade Federal Rural do Semi-Árido. MossoróRN,Brasil.karolinesoares@ufersa.edu.br

${ }^{3}$ Professor do Centro de Ciências Agrárias, Universidade Federal Rural do Semi Arido. Mossoró-RN, Brasil. vilsongois@ufersa.edu.br

${ }^{4}$ Mestrando em Ciência Animal. Centro de Ciências Agrárias, Universidade Federal Rural do Semi-Arido. Mossoró-RN, Brasil. clevtonazevedo@hotmail.com

${ }^{5}$ Discente do curso de graduação em Biotecnologia, Universidade Federal Rural do Semi-Árido. Mossoró-RN, Brasil. danielarsmorais@hotmail.com

${ }^{6}$ Biotecnologista, Universidade Federal Rural do Semi-Árido. Mossoró-RN, Brasil. amanda-sabino@hotmail.com

*Autor para correspondência

RESUMO. O pescado é bastante consumido mundialmente, dentre inúmeros motivos, por ser um alimento rico em proteínas e aminoácidos essenciais à saúde humana, destacandose como um atraente produto de origem animal. No entanto, devido à alta perecibilidade que apresenta, o produto deve receber atenção especial. Dessa forma, o presente estudo teve por objetivo avaliar a influência do ácido cítrico e lactato de sódio sobre as características físico-químicas da Carapeba (Diapterus rhombeus) minimamente processada. As amostras foram coletadas em uma feira-livre localizada no município de Mossoró-RN e seguiram ao laboratório onde foram submetidas ao processo de filetagem. Os filés produzidos receberam tratamentos distintos: ausência de imersão em acidulantes (controle) e imersão em solução de ácido cítrico e lactato de sódio. As amostras de ambos os tratamentos foram embaladas a vácuo, aos pares, e estocadas em refrigeração. Durante o armazenamento, foram realizadas análises físico-químicas nos dias zero, três e seis, em três repetições por tratamento. Os dados gerados foram avaliados pelo teste $t$ studant ao nível de $5 \%$ de significância. Com a utilização do tratamento, observou-se resultados satisfatórios de capacidade de retenção de líquido e $\mathrm{pH}$ nos filés de peixe minimamente processados e embalados a vácuo sob refrigeração.

Palavras chave: Conservação, embalagem modificada, pescado, qualidade físico-química

\section{Quality aspects physico-chemical in fillet minimally processed Diapterus rhombeus}

ABSTRACT. Fish is widely consumed worldwide, among many reasons, being a food rich in proteins and amino acids essential to human health, standing out as an attractive product of animal origin. However, due to the high perishability it presents, the product should receive special attention. Thus, the present study aimed to evaluate the influence of citric acid and sodium lactate on the physicochemical characteristics of the minimally processed Diapterus rhombeus. The samples were collected in a fair-free located in the municipality of Mossoró-RN and followed the laboratory where they were submitted to the filleting process. The fillets produced received different treatments: absence of immersion in acidulants (control) and immersion in citric acid solution and sodium lactate. Samples from both treatments were vacuum packed in pairs and stored under refrigeration. During 
storage, physical and chemical analyzes were performed at days zero, three and six, in three replicates per treatment. The data generated were evaluated by the $t$ student test at the $5 \%$ level of significance. With the use of the treatment, satisfactory results of CRA and $\mathrm{pH}$ were observed in minimally processed fish fillets and vacuum packaged under refrigeration.

Keywords: Conservation, modified packaging, fish, physical-chemical quality

\section{Aspectos de calidad fisicoquímica en filete de Diapterus rhombeus mínimamente procesado}

RESUMEN. El pescado es bastante consumido mundialmente, de entre innumerables motivos, por ser un alimento rico en proteínas y aminoácidos esenciales a la salud humana, destacándose como un atractivo producto de origen animal. Sin embargo, debido a la alta perecibilidad que presenta, el producto debe recibir atención especial. De esta forma, el presente estudio tuvo como objetivo evaluar la influencia del ácido cítrico y lactato de sodio sobre las características fisicoquímicas del filete de Diapterus Rhombeus mínimamente procesado. Las muestras fueron recolectadas en una feria libre localizada en el municipio de Mossoró-RN y siguieron al laboratorio donde fueron sometidas al proceso de fileteado. Los filetes producidos recibieron tratamientos distintos: ausencia de inmersión en acidulantes (control) e inmersión en solución de ácido cítrico y lactato de sodio. Las muestras de ambos tratamientos fueron envasadas al vacío, en pares, y refrigeradas. Durante el almacenamiento, fueron realizadas análisis fisicoquímicos en los días cero, tres y seis, en tres repeticiones por tratamiento. Los datos generados fueron evaluados por la prueba $t$ de Studant al nivel de 5\% de significancia. Con la utilización del tratamiento, se observó resultados satisfactorios de capacidad de retención de agua y $\mathrm{pH}$ en los filetes de pescado mínimamente procesados y envasados al vacío bajo refrigeración.

Palabras clave: conservación, envase modificado, pescado, calidad fisicoquímica

\section{Introdução}

Os hábitos alimentares têm mudado conforme o ritmo de vida da população, que atualmente busca produtos mais saudáveis e, entre outros, que proporcione segurança ao consumidor. Para atender tal necessidade, os alimentos minimamente processados são vistos como uma alternativa (Freire et al., 2015, Oetterer et al., 2006, Santos and Oliveira, 2012). Dentre os alimentos enquadrados nesta categoria, tem-se o pescado, cujo consumo está associado à qualidade de vida (Sartori and Amancio, 2012).

O termo "Pescado" compreende todo organismo aquático destinado à alimentação humana, podendo ser consumido diretamente ou aproveitado na indústria (Barros, 2003).

Com o aumento da demanda, a produção do pescado tem ganhado força e recebido grandes investimentos (Oetterer et al., 2006). No entanto, sua alta perecibilidade acaba por afetar a comercialização, sendo a manipulação e o tempo de captura até a chegada ao consumidor, fortes determinantes do grau de alteração que sofrerá (Ordóñez, 2005). Assim, tem-se que sua vida útil dependerá do método de conservação utilizado, e este precisa preservar ao máximo as características do mesmo (Neiva et al., 2015).

Alguns ácidos orgânicos e seus sais estão presentes naturalmente em carnes de pescado e outras, podendo ser utilizados como aditivo (Freire et al., 2016, Theron and Lues, 2007). Os aditivos vêm demonstrando eficiência sobre parâmetros de qualidade de pescado minimamente processado, proporcionando a sua conservação (Freire et al., 2016, Freire et al., 2015).

Apesar dos benefícios aparentes da aplicação de tais ácidos em alimentos (Freire et al., 2016, Freire et al., 2015, Silva et al., 2014), a interferência sobre as características físicoquímicas destes precisa receber maior investigação, já que as propriedades funcionais dos alimentos podem ser comprometidas pelo processo.

Com base no que foi exposto, o presente estudo teve por objetivo avaliar a influência do lactato de sódio e ácido cítrico nas propriedades físicoquímicas do peixe Carapeba minimamente processado. 


\section{Material e Métodos}

Para o seguinte estudo utilizou-se peixes do tipo Carapeba (Diapterus rhombeus), obtidos de feira local no município de Mossoró-RN. Estes foram devidamente acondicionados em caixas isotérmicas, contendo gelo de qualidade, para transporte até o Laboratório de Biotecnologia Industrial (LABIND), localizado na Universidade Federal Rural do Semi Árido (UFERSA), onde foram realizadas as análises.

As amostras foram colocadas em bandejas e homogeneizadas. Em seguida, com o auxílio de bisturis estéreis, realizou-se a filetagem (retirada dos filés). Foram obtidos, por amostra, pares de filés com pele, correspondentes a cada lado do peixe. Na etapa seguinte, os filés foram colocados em bandeja plástica, lavados com água corrente para retirada de sujidades e separados em dois lotes para aplicação de tratamentos distintos: TC (Controle) e o TA (Tratamento com solução dos aditivos lactato de sódio $3 \%$ e ácido cítrico $1 \%$ ). As amostras do TC e TA foram dispostas em bandejas forradas com camadas de papel toalha, previamente esterilizado por radiação ultravioleta, para a retirada do excesso de umidade. Para os filés do TA, realizou-se a imersão em solução de ácido cítrico e lactato de sódio por 5 minutos, com viragem a cada 2 minutos e 30 segundos. Com a finalidade de remover a solução presente em demasia nas amostras, as mesmas foram suspensas por meio metro e logo dispostas em bandejas forradas com camadas de papel toalha, conforme metodologia descrita por Soares et al. (2016).

Foram obtidos 18 pares de filés para ambos os tratamentos, acondicionados, aos pares, em sacos plásticos impermeáveis ao oxigênio e embalados a vácuo. As amostras foram armazenadas sob refrigeração, simultaneamente, com temperaturas entre 4 e $7^{\circ} \mathrm{C}$.

Realizaram-se análises nos dias zero, três e seis, em intervalos regulares de 72 horas. Os parâmetros utilizados na análise foram: potencial hidrogênio $(\mathrm{pH})$, perda por exsudação (PE), capacidade de retenção de líquido (CRA), perda de peso por cocção (PPC) e cor ( $a^{*}, b^{*}$ e $\left.L^{*}\right)$.

Para a análise de $\mathrm{pH}$, utilizou-se potenciômetro digital, conforme citado na metodologia proposta pelo Instituto Adolfo Lutz (Lutz, 2005). A PE foi aferida segundo a proposta de Caldara et al. (2012) modificada, e o valor para perda de exsudato foi expresso pelo percentual de água perdida em relação ao peso de $100 \mathrm{~g}$ da amostra. Seguindo a metodologia descrita por Sanfelice et al. (2010) foram mensurados os valores para CRA, PPC e cor.

$\mathrm{Na}$ medição da cor, foram conferidos os parâmetros $\mathrm{L}^{*}$ (luminosidade), $\mathrm{a}^{*}$ (teor de vermelho) e $b^{*}$ (teor de amarelo) através da utilização de um colorímetro digital portátil. Nessa análise, os filés foram dispostos em uma superfície plana submetida a iluminação exclusivamente artificial e as aferições foram realizadas em três regiões distintas dos mesmos, no lado com pele, posteriormente no lado sem pele. O resultado final foi obtido através da média das três leituras realizadas para cada lado.

O experimento foi conduzido através de delineamento inteiramente casualizado com dois tratamentos e três repetições. Os dados das análises físico-químicas foram submetidos ao teste de $\mathrm{t}$ Student para a comparação das médias, ao nível de 5\% de significância.

\section{Resultados e Discussão}

Os valores de $\mathrm{pH}$ para a amostra controle no dia 0,3 e 6, foram de: 7,27, 7,22 e 7,17, respectivamente (Tabela 1). De mesmo modo, os valores das amostras tratadas para os dias seguidos: 6,38, 6,71 e 6,55. Ao analisar, constatou-se que a amostra controle, independente do dia de estocagem, mostrou valores mais elevados de $\mathrm{pH}$ em relação a tratada, e diferenças, estatisticamente significativas, ao terceiro dia.

Tal fato pode ser decorrente da absorção de ácido pelas amostras tratadas, onde um ácido ao se ionizar libera prótons $\mathrm{H}^{+}$, que interferem diretamente no $\mathrm{pH}$, no qual é dado pela expressão: $-\log \left[\mathrm{H}^{+}\right]$. A utilização da solução de lactato de sódio e ácido cítrico, possivelmente, resultou em um decréscimo dos valores do $\mathrm{pH}$ no tempo 3, tornando o produto mais ácido, possibilitando um maior tempo de conservação (Tavares and Gonçalves, 2011).

Para PE não existem valores de perda para o dia 0 , pois este parâmetro só é possível analise após prévia refrigeração. Para os dias três e seis, a amostra controle apresentou PE de 2,80\% e $3,02 \%$, e a amostra tratada, $0,76 \%$ e $3,85 \%$ (Tabela 1). Observou-se uma significativa diferença entre a amostra tratada e a controle no terceiro dia. Os resultados maiores de PE nas amostras foram possivelmente devido ao ácido do tratamento, no qual funcionou como um eletrólito, retirando a água do músculo do peixe, reduzindo o pH da amostra, aproximando-o do $\mathrm{pH}$ 
isoelétrico, onde é menor a capacidade de ligação de água pelas proteínas.

Tabela 1. Valores obtidos para as variáveis físico-químicas no controle e tratamento com aditivos orgânicos em peixe Carapeba minimamente processado durante os seis dias de armazenamento.

\begin{tabular}{lcccccc}
\hline \multirow{2}{*}{ Análises } & \multicolumn{3}{c}{ Dia 0} & \multicolumn{2}{c}{ Dia 3} & \multicolumn{2}{c}{ Dia 6} \\
\cline { 2 - 7 } & Controle & Tratado & Controle & Tratado & Controle & Tratado \\
\hline $\mathrm{pH}$ & $7,27^{\mathrm{a}}$ & $6,38^{\mathrm{a}}$ & $7,22^{\mathrm{a}}$ & $6,71^{\mathrm{b}}$ & $7,17^{\mathrm{a}}$ & $6,55^{\mathrm{a}}$ \\
$\mathrm{PE}$ & - & - & $2,80^{\mathrm{a}}$ & $0,76^{\mathrm{a}}$ & $3,02^{\mathrm{a}}$ & $3,85^{\mathrm{b}}$ \\
$\mathrm{CRA}, \%$ & $60^{\mathrm{a}}$ & $43,97^{\mathrm{a}}$ & $47,45^{\mathrm{a}}$ & $40,87^{\mathrm{a}}$ & $49,78^{\mathrm{a}}$ & $34,18^{\mathrm{b}}$ \\
PPC, \% & $19,47^{\mathrm{a}}$ & $30,18^{\mathrm{b}}$ & $38,26^{\mathrm{a}}$ & $36,20^{\mathrm{b}}$ & $36,13^{\mathrm{a}}$ & $40,36^{\mathrm{b}}$ \\
$\mathrm{L}^{*}$ & $34,27^{\mathrm{a}}$ & $41,97^{\mathrm{b}}$ & $44,52^{\mathrm{a}}$ & $51,03^{\mathrm{a}}$ & $54,80^{\mathrm{a}}$ & $50,74^{\mathrm{b}}$ \\
$\mathrm{a}^{*}$ & $2,33^{\mathrm{a}}$ & $2,77^{\mathrm{b}}$ & $3,08^{\mathrm{a}}$ & $3,08^{\mathrm{b}}$ & $4,20^{\mathrm{a}}$ & $3,35^{\mathrm{b}}$ \\
$\mathrm{b}^{*}$ & $2,33^{\mathrm{a}}$ & $2,77^{\mathrm{b}}$ & $2,09^{\mathrm{a}}$ & $0,95^{\mathrm{b}}$ & $2,63^{\mathrm{a}}$ & $1,41^{\mathrm{b}}$ \\
\hline
\end{tabular}

${ }^{\mathrm{a}, \mathrm{b}}$ Letras diferentes entre os dois tratamentos referente a um determinado dia de avaliação, indica diferença significativa no teste de t Student ao nível de 5\% de probabilidade. L*: Luminosidade; a*: Teor de Vermelho; b*: Teor de Amarelo.

$\mathrm{Na}$ CRA, o controle apresentou os valores $60 \%, 47,45 \%$ e $49,78 \%$ nos dias 0,3 e 6 e a tratada: $\quad 43,97 \%, \quad 40,87 \% \quad$ e $\quad 34,18 \%$, respectivamente (Tabela 1). Os valores inferiores no grupo tratamento tornaram evidente uma maior capacidade de retenção da umidade do peixe, com diferença significativa ao sexto dia de análise. O resultado obtido, possivelmente, se deu pela presença de sal, que promove a maior solubilidade das proteínas aumentando a sua retenção de água (Belitz and Grosch, 1997, Roque-Specht et al., 2009).

Para a análise de PPC, o valor da amostra controle do dia 0 foi de $19,47 \%$ e a controle, $30,18 \%$. No dia 3, o controle foi de $38,26 \%$ e o tratado, $36,20 \%$. No último dia de estocagem os valores apresentados para os tratamentos controle e tratado foram, respectivamente, $36,13 \%$ e $40,36 \%$. A cocção é um processo no qual ocorrem trocas químicas, físico-químicas e estruturais dos componentes dos alimentos, que se dão pela aplicação do calor, sendo os fatores temperatura, duração do processo e meio de cocção, essenciais à determinação dessas mudanças (Rosa et al., 2006). Em todos os dias, a PPC mostrou distinção estatística nas amostras controle e tratada, mas pelo aumento observado no parâmetro CRA com o tratamento, é bem possível que as perdas geradas pelo processo de cocção, de um modo geral, estejam relacionadas a perda de gordura, não de umidade, já que o processo altera além de teores de proteína e cinzas, também de gordura.

No parâmetro cor, as luminosidades $\left(\mathrm{L}^{*}\right)$ detectadas nas amostras controle ao longo da estocagem foram: $34,27,44,52$ e 54,80 , enquanto que no tratado foram encontrados os seguintes resultados: 41,97, 51,03 e 50,74. Para teor de vermelho $\left(a^{*}\right)$, a amostra controle do dia $(0)$ teve como resultado 2,33 e a controle, 2,77 , para o dia (3), 3,08 e 3,08, no controle e tratada, respectivamente. No dia (6), o controle foi de 4,20 e no tratado 3,35. O índice de amarelo (b*) apresentou nas amostras controle os valores: 2,33 , 2,09 e 2,63, respectivamente. Para a amostra tratada: $2,77,0,95$ e 1,41 . Desse modo foram encontradas diferenças estatísticas entre amostra controle e tratada tanto para luminosidade, quanto para os teores de vermelho e amarelo. Foi observado que o teor de vermelho apresentou aumento com o tratamento ao primeiro dia e ocorreu um decréscimo deste durante a estocagem. Já para o amarelo, seu valor aumentou, sofreu uma queda com a estocagem e, em seguida, se elevou novamente. De modo geral, a cor se mostrou um parâmetro positivo quanto à atividade do tratamento.

\section{Conclusão}

Dessa forma, a produção da Carapeba minimamente processada associando a utilização de solução de lactato de sódio e ácido cítrico, antes da embalagem a vácuo, para posterior estocagem, se mostrou eficiente sobre alguns de seus aspectos físico-químicos, tais como a capacidade de retenção de água, cor e $\mathrm{pH}$.

\section{Referências bibliográficas}

Barros, G. C. 2003. Perda de qualidade do pescado, deterioração e putrefação. Revista do Conselho Federal de Medicina Veterinária, 30, 59-64.

Belitz, H. D. \& Grosch, W. 1997. Química de los alimentos. Acribia, Zaragoza.

Caldara, F. R., Santos, V. M. O., Santiago, J. C., Almeida Paz, I. C. d. L., Garcia, R. G., Vargas Junior, F. M., Santos, L. S. \& Nääs, I. d. A. 
2012. Physical and sensory properties of PSE pork. Revista Brasileira de Saúde e Produção Animal, 13, 815-824.

Freire, B. C. F., Paiva Soares, K. M., Azevedo Costa, A. C. A., Souza, A. S., Silva, L. K. C., Góis, V. A., Bezerra, A. C. D. S. \& Gomes, H. A. N. 2016. Qualidade de camarão (Litopenaeus vannamei) minimamente processado. Acta Veterinaria Brasilica, 10, 150-155.

Freire, B. C. F., Paiva Soares, K. M., Souza, A. S., Azevedo, A. C. A. \& Góis, V. A. 2015. Staphylococcus spp. em camarão minimamente processado refrigerado embalado a vácuo. Revista Verde de Agroecologia e Desenvolvimento Sustentável, 10, 84-87.

Lutz, N. A. d. I. A. 2005. Métodos químicos e físicos para análise de alimentos 4th ed. São Paulo, Brazil.

Neiva, C. R. P., Matsuda, C. S., Machado, T. M., Casarini, L. M. \& Tomita, R. Y. 2015. Glaciamento em filé de peixe congelado: revisão dos métodos para determinação de peso do produto. Boletim do Instituto de Pesca, 41, 899-906.

Oetterer, M., D'Arce, M. A. B. R. \& Spoto, M. 2006. Fundamentos de ciencia e tecnologia de alimentos. Editora Manole Ltda, Barueri - São Paulo.

Ordóñez, J. A. 2005. Tecnologia de alimentos. Artmed, Porto Alegre.

Roque-Specht, V. F., Simoni, V., Parise, N. \& Cardoso, P. G. 2009. Avaliação da capacidade de retenção de água em peitos de frango em função do pH final. Current Agricultural Science and Technology, 15, 77-81.

Rosa, F. C., Bressan, M. C., Bertechini, A. G., Fassani, E. J., Vieira, J. O., Faria, P. B. \& Savian, T. V. 2006. Efeito de métodos de cocção sobre a composição química e colesterol em peito e coxa de frangos de corte. Revista Ciência e Agrotecnologia, 30, 707714.
Sanfelice, C., Mendes, A. A., Komiyama, C. M., Cañizares, M. C., Rodrigues, L., Cañizares, G. I., Roça, R. O., Almeida, I. C. L. P., Balog, A. \& Milbradt, E. L. 2010. Avaliação e caracterização da qualidade da carne de peito (Pectoralis major) de matrizes pesadas em final de ciclo produtivo. Food Science and Technology, 30, 166-170.

Santos, J. S. \& Oliveira, M. B. P. P. 2012. Revisão: alimentos frescos minimamente processados embalados em atmosfera modificada. Brazilian Journal of Food Techonoly, 15, 1-14.

Sartori, A. G. O. \& Amancio, R. D. 2012. Pescado: importância nutricional e consumo no Brasil. Segurança Alimentar e Nutricional, 19, 83-93.

Silva, R. X. A., José, K. F. C., Franco, R. M. \& Silva, T. J. P. 2014. Lactato de sódio, nisina e sua combinação na validade comercial da linguiça Toscana embalada a vácuo e estocada a $4^{\circ} \mathrm{C}$. Ciência Rural, 44, 746-751.

Soares, K. M. P., Souza, L. B. \& Silva, J. B. A. 2016. Coliformes totais e termotolerantes em bifes de carne bovina tratados com ácido lático e lactato de sódio. Revista Brasileira de Ciência Veterinária, 23, 196-199.

Tavares, M. \& Gonçalves, A. A. 2011. Aspectos físico-químicos do pescado. In: Gonçalves, A. A. (ed.) Tecnologia do Pescado. Atheneu, São Paulo.

Theron, M. M. \& Lues, J. F. R. 2007. Organic acids and meat preservation: a review. Food Reviews International, 23, 141-158.

\section{Article History:}

Received 26 January 2017

Accepted 25 April 2017

Available on line 18 May 2017

License information: This is an open-access article distributed under the terms of the Creative Commons Attribution License 4.0, which permits unrestricted use, distribution, and reproduction in any medium, provided the original work is properly cited 